\title{
Classification of water molecules in protein binding sites.
}

\author{
Caterina Barillari, ${ }^{\dagger}$ Justine Taylor, ${ }^{\dagger}$ Russell Viner ${ }^{\ddagger}$ and Jonathan W. Essex ${ }^{*} \dagger$ \\ ${ }^{\dagger}$ School of Chemistry, University of Southampton, Highfield, Southampton, SO17 1BJ, UK \\ ${ }_{\ddagger}^{\ddagger}$ Syngenta, Jealott’s Hill International Research Centre, Bracknell, RG42 6EY, UK
}

E-mail: J.W.Essex@ soton.ac.uk

\section{System preparation}

HIV-1 protease. His 69 and His 69' were protonated on N $\delta$. Asp 25 and Asp 25' were both protonated in complexes lecl, lec0, lebw, leby; in complex 1hpx Asp 25 was protonated and Asp 25' was deprotonated. The total charge of the protein is $+5 \mathrm{e}$ for 1 hpx and $+6 \mathrm{e}$ for the other complexes. All inhibitors are neutral. Electrostatic neutrality was guaranteed by adding 5 chlorine ions to the $1 \mathrm{hpx}$ complex and 6 chlorine ions to the other complexes.

Neuraminidase. The following tautomers were adopted for histidines: His 98, 150, 233 and 312 were protonated on $\mathrm{N} \delta$, while His 144, 184, 274 were protonated on $N \varepsilon$. The net charge of the protein is $-3 \mathrm{e}$. All the crystallographic structures considered contain sugars, but they were not included in the set up of the systems. The structures also contain two calcium ions: one is located inside a cavity of the protein and it was retained as it is structurally important; ${ }^{1}$ the other ion is outside the protein and it was discarded. The inhibitors have the following total charges: DANA (pdb 1f8b) and sialic acid (pdb Imwe) are charged -1e, while all the other inhibitors have a total charge of zero. Electrostatic neutrality of the systems was guaranteed by adding two sodium ions to the complexes in $1 f 8 b$ and $1 m w e$ and one sodium ion to the other complexes, using $L E a P$ in the Amber 7 package.

Trypsin. The histidine residue in the active site (His 57) is known to be charged, ${ }^{2}$ while the other two histidines (His 40 and His 91) were considered to be protonated on Ne. The net charge of the protein is +7 e. Inhibitors have the following total charges: $+2 \mathrm{e}$ for ligands in complexes $1 a z 8$ and $1 f O u,+1 \mathrm{e}$ for the remaining ligands. The calcium ion present in the majority of the structures is important to stabilise the structure and, as such, it was retained. In the complex $1 a z 8$ the ion was not originally present in 
the structure and a water molecule was found in its position; in this case the water was replaced by a calcium ion. Electrostatic neutrality of each system was guaranteed by adding the correct number of chlorine ions with $L E a P$ : 11 chlorine ions were added in complexes $1 a z 8$ and $1 f 0 u ; 10$ ions were added in the other complexes.

Factor Xa. For histidine residues the following protonation state was adopted: His 13, 91, 199 were protonated on $\mathrm{N} \varepsilon$ and His $57,83,145$ were protonated on $\mathrm{N} \delta$. The heavy chain of the protein (chain A) has a net charge of $+1 \mathrm{e}$, while the light chain (chain B) has a net charge of -2e. One calcium ion is present in all the structures and, since it is structurally important, it was retained. ${ }^{3}$ The following charges were adopted for the ligands: $+2 \mathrm{e}$ for inhibitor in complexes 1 ezq and $1 \mathrm{lpg} ;+1 \mathrm{e}$ for the remaining three inhibitors. Electrostatic neutrality of the systems was guaranteed by adding three chlorine ions in the complexes 1 ezq and llpg and two chlorine ions in the other complexes using the LEaP program in the Amber 7 package.

Scytalone dehydratase. The enzyme in pdb entry 3std misses two residues: Asp 171 and Lys 172. The two residues were added using the structure of $7 s t d$ as a template. The two histidine residues in the binding site (His 85 and His 110) are known to be protonated on the $\mathrm{N} \delta ;^{4}$ for His 104, 128, 133 the $\mathrm{N} \delta$ tautomer was adopted while for His 126 the $\mathrm{N} \varepsilon$ tautomer was adopted. Each monomer in the protein has a net charge of $-4 \mathrm{e}$, thus resulting in a total charge of the protein of -12e. All the inhibitors considered are neutral. Electrostatic neutrality of the systems was ensured by adding twelve sodium ions with the program $L E a P$ in the Amber 7 package.

OppA. In five of the six structures considered only one chain is present, while in pdb entry $l b 3 l$ there are two chains. In this case, only one chain (chain A in the pdb) was modelled and only crystallographic water molecules within $10 \AA$ of it were retained. The uranium and acetate ions present in some of the structures were discarded. Histidine residues were considered to be monoprotonated and the following tautomers were adopted: His 55, 75, 117, 317, 517 were protonated on $\mathrm{N} \delta$ while His 29, 91, 142, $161,405,440$ were protonated on $\mathrm{N} \varepsilon$. The protein has a net charge of -8e. Inhibitors have different total charges: KGK, KWK and KAK (respectively pdb 1 b3l, ljev, ljet) are charged +2e; KDK and KEK (respectively pdb $1 b 4 z, 1$ jeu $)$ are charged $+1 \mathrm{e}$ and KKK (pdb 2olb) is charged $+3 \mathrm{e}$. Electrostatic neutrality of the systems was ensured by adding the correct number of sodium ions with the program 
$L E a P$ in the Amber 7 package: six ions were added to $1 \mathrm{~b} 3 \mathrm{l}, 1 \mathrm{jev}, 1 \mathrm{jet}$; seven ions were added to $1 \mathrm{~b} 4 \mathrm{z}$ and ljeu; five ions were added to $2 o l b$.

\section{Free energy gradients as a function of lambda}

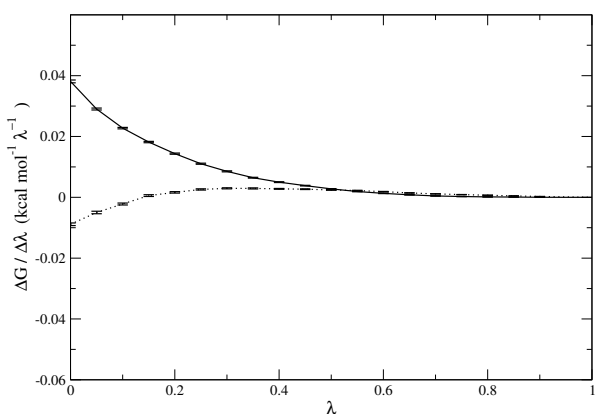

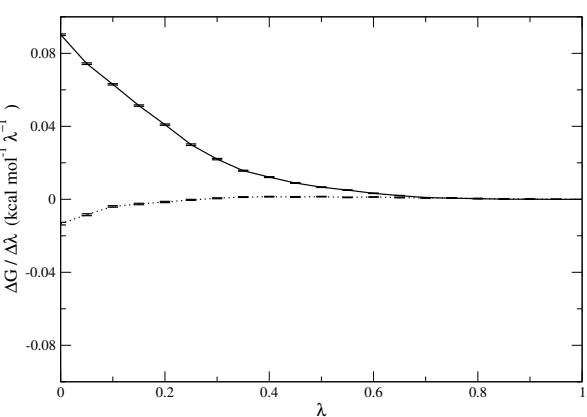

$\mathrm{b}$

Figure 1. Free energy gradients as a function of the coupling parameter $\lambda$ for (a) Wat 1401 in $1 \mathrm{f} 8 \mathrm{~b}$ and (b) Wat 2221 in 1f8b. The thick line represents the electrostatic decoupling; the dotted line represents the Lennard-Jones decoupling.

\section{Complete References 24 and 26}

(24) von Itzstein, M.; Wu, W-Y.; Kok, G. B.; Pegg, M. S.; Dyason, J. C.; Jin, B.; Phan, T. V.; Smythe, M. L.; White, H. F.; Oliver, S. W. ; Colman, P. M.; Varghese, J. N.; Ryan, D. M.; Woods, J. M.; Bethell, R. C.; Hotham, V. J.; Cameron, J. M.; Penn, C. R. Nature, 1993, 363, 418-423.

(26) Guertin, K. R.; Gardner, C. J.; Klein, S. I.; Zulli, A. L.; Czekaj, M.; Gong, Y.; Spada, A. P.; Cheney, D. L.; Maignan, S.; Guilloteau, J. P.; Brown, K. D.; Colussi, D. J.; Chu, V.; Heran, C. L.; Morgan, S. R.; Bentley, R. G.; Dunwiddie, C. T.; Leadley, R. J.; Pauls, H. W. Bioorg. Med. Chem. Lett., 2002, 12, 1671-1674. 


\section{Waters and ligands free energies}

Table 1 shows the data used to generate figure 10 in the paper: $\Delta G_{w a t}$ is the calculated binding free energy of the water and $\Delta \Delta G_{\text {lig }}$ is the experimental relative binding free energy of ligands.

TABLE 1: Free energies of waters and ligands

\begin{tabular}{|c|c|c|c|c|c|}
\hline$p d b(\text { water })^{a}$ & $\Delta G_{w a t}^{b}$ & $\Delta G_{l i g 1}^{c}$ & Ligand $2^{d}$ & $\Delta G_{\text {lig2 }}^{e}$ & $\Delta \Delta G_{\text {lig }}^{f}$ \\
\hline $1 \mathrm{f} 8 \mathrm{~b}(1401)$ & 0.9 & $-7.4^{5}$ & $117 \mathrm{f}$ & $-14.8^{6}$ & -7.4 \\
\hline $1 \mathrm{f} 8 \mathrm{~b}(1401)$ & 0.9 & -7.4 & $1 \mathrm{nnc}$ & $-12.3^{7}$ & -4.9 \\
\hline $1 \mathrm{f} 8 \mathrm{~b}(1401)$ & 0.9 & -7.4 & 2qwi & $-7.7^{8}$ & -0.3 \\
\hline $1 \mathrm{f} 8 \mathrm{c}(1001)$ & 1.7 & $-10.1^{5}$ & $117 \mathrm{f}$ & -14.8 & -4.7 \\
\hline $1 \mathrm{f} 8 \mathrm{c}(1001)$ & 1.7 & -10.1 & $1 \mathrm{nnc}$ & -12.3 & -2.2 \\
\hline $1 \mathrm{f} 8 \mathrm{c}(1001)$ & 1.7 & -10.1 & 2qwi & -7.7 & 2.4 \\
\hline $1 \mathrm{mwe}(328)$ & 0.7 & $-3.0^{9}$ & $117 \mathrm{f}$ & -14.8 & -11.8 \\
\hline $1 \mathrm{mwe}(328)$ & 0.7 & -3.0 & $1 \mathrm{nnc}$ & -12.3 & -9.3 \\
\hline $1 \mathrm{mwe}(328)$ & 0.7 & -3.0 & 2qwi & -7.7 & -4.7 \\
\hline $2 q w j(8)$ & 0.8 & $-12.8^{7}$ & $117 \mathrm{f}$ & -14.8 & -12.0 \\
\hline $2 q w j(8)$ & 0.8 & -12.8 & $1 \mathrm{nnc}$ & -12.3 & 0.5 \\
\hline 2qwj(8) & 0.8 & -12.8 & 2qwi & -7.7 & 5.1 \\
\hline $2 q w k(2)$ & 2.3 & $-13.6^{6}$ & $117 \mathrm{f}$ & -14.8 & -1.2 \\
\hline $2 \mathrm{qwk}(2)$ & 2.3 & -13.6 & $1 \mathrm{nnc}$ & -12.3 & 1.3 \\
\hline $2 q w k(2)$ & 2.3 & -13.6 & 2qwi & -7.7 & 5.9 \\
\hline $1 \mathrm{hpx}(301)$ & -10.0 & $-15.4^{10}$ & $1 q b s$ & $-13.0^{11}$ & 2.4 \\
\hline $1 \mathrm{hpx}(301)$ & -10.0 & -15.4 & $1 \mathrm{dmp}$ & $-13.0^{12}$ & 2.4 \\
\hline $1 \mathrm{hpx}(301)$ & -10.0 & -15.4 & $1 \mathrm{hwr}$ & $-11.3^{13}$ & 4.1 \\
\hline $1 \mathrm{hpx}(301)$ & -10.0 & -15.4 & 1ajx & $-10.8^{14}$ & 4.6 \\
\hline $1 \mathrm{hpx}(301)$ & -10.0 & -15.4 & $1 \mathrm{hvr}$ & $-13.0^{11}$ & 2.4 \\
\hline $1 \mathrm{hpx}(301)$ & -10.0 & -15.4 & $1 \mathrm{bv} 7$ & $-12.7^{15}$ & 2.7 \\
\hline $1 \mathrm{ec} 1(614)$ & -9.7 & $-12.2^{16}$ & $1 \mathrm{qbs}$ & -13.0 & -0.8 \\
\hline $1 \mathrm{ec} 1(614)$ & -9.7 & -12.2 & $1 \mathrm{dmp}$ & -13.0 & -0.8 \\
\hline 1ec1(614) & -9.7 & -12.2 & 1hwr & -11.3 & 0.8 \\
\hline $1 \mathrm{ec} 1(614)$ & -9.7 & -12.2 & 1ajx & -10.8 & 1.4 \\
\hline 1ec1(614) & -9.7 & -12.2 & $1 \mathrm{hvr}$ & -13.0 & -0.8 \\
\hline 1ec1(614) & -9.7 & -12.2 & 1bv7 & -12.7 & -0.5 \\
\hline $1 \mathrm{ec} 0(619)$ & -8.2 & $-12.0^{17}$ & $1 \mathrm{qbs}$ & -13.0 & -1.0 \\
\hline $1 \mathrm{ec} 0(619)$ & -8.2 & -12.0 & $1 \mathrm{dmp}$ & -13.0 & -1.0 \\
\hline $1 \mathrm{ec} 0(619)$ & -8.2 & -12.0 & 1hwr & -11.3 & 0.7 \\
\hline
\end{tabular}




\begin{tabular}{|c|c|c|c|c|c|}
\hline$p d b(\text { water })^{a}$ & $\Delta G_{\text {wat }}^{b}$ & $\Delta G_{l i g 1}^{c}$ & Ligand $^{d}$ & $\Delta G_{\text {lig2 }}^{e}$ & $\Delta \Delta G_{l i g}^{f}$ \\
\hline $1 \mathrm{ec} 0(619)$ & -8.2 & -12.0 & 1ajx & -10.8 & 1.2 \\
\hline $1 \mathrm{ec} 0(619)$ & -8.2 & -12.0 & $1 \mathrm{hvr}$ & -13.0 & -1.0 \\
\hline $1 \mathrm{ec} 0(619)$ & -8.2 & -12.0 & 1bv7 & -12.7 & -0.7 \\
\hline 1ebw(319) & -7.7 & $-12.4^{18}$ & $1 \mathrm{qbs}$ & -13.0 & -0.6 \\
\hline 1ebw(319) & -7.7 & -12.4 & $1 \mathrm{dmp}$ & -13.0 & -0.6 \\
\hline 1ebw(319) & -7.7 & -12.4 & 1hwr & -11.3 & 1.1 \\
\hline 1ebw(319) & -7.7 & -12.4 & 1 ajx & -10.8 & 1.6 \\
\hline 1ebw(319) & -7.7 & -12.4 & $1 \mathrm{hvr}$ & -13.0 & -0.6 \\
\hline 1ebw(319) & -7.7 & -12.4 & $1 \mathrm{bv} 7$ & -12.7 & -0.3 \\
\hline 1eby(316) & -7.1 & $-12.2^{17}$ & 1qbs & -13.0 & -0.8 \\
\hline 1eby(316) & -7.1 & -12.2 & $1 \mathrm{dmp}$ & -13.0 & -0.8 \\
\hline 1eby(316) & -7.1 & -12.2 & 1hwr & -11.3 & 0.8 \\
\hline 1eby(316) & -7.1 & -12.2 & 1ajx & -10.8 & 1.4 \\
\hline 1eby(316) & -7.1 & -12.2 & $1 \mathrm{hvr}$ & -13.0 & -0.9 \\
\hline 1eby(316) & -7.1 & -12.2 & 1bv7 & -12.7 & -0.5 \\
\hline $4 \operatorname{std}(64)$ & -3.1 & $-14.0^{19}$ & 3std & $-15.2^{20}$ & -1.2 \\
\hline $5 \operatorname{std}(537)$ & -5.7 & $-14.3^{19}$ & 3 std & -15.2 & -0.9 \\
\hline $6 \operatorname{std}(57)$ & -5.5 & $-11.8^{19}$ & 3 std & -15.2 & -3.4 \\
\hline $7 \operatorname{std}(91)$ & -5.0 & $-14.6^{19}$ & 3 std & -15.2 & -0.6 \\
\hline 1ezq(100) & -4.4 & $-12.3^{21}$ & $1 \mathrm{mq} 5$ & $-12.3^{22}$ & 0.0 \\
\hline lezq(100) & -4.4 & -12.3 & $1 \mathrm{mq} 6$ & $-13.8^{22}$ & -1.5 \\
\hline 1ezq(100) & -4.4 & -12.3 & $1 \mathrm{nfw}$ & $-12.2^{23}$ & 0.1 \\
\hline lezq(100) & -4.4 & -12.3 & 1nfu & $-10.6^{23}$ & 1.7 \\
\hline 1ezq(100) & -4.4 & -12.3 & $1 \mathrm{nfx}$ & $-11.6^{23}$ & 0.7 \\
\hline lezq(100) & -4.4 & -12.3 & 1nfy & $-12.1^{23}$ & 0.2 \\
\hline $1 \mathrm{ksn}(16)$ & -3.7 & $-12.8^{24}$ & $1 \mathrm{mq} 5$ & -12.3 & 0.5 \\
\hline $1 \mathrm{ksn}(16)$ & -3.7 & -12.8 & $1 \mathrm{mq} 6$ & -13.8 & -1.0 \\
\hline $1 \mathrm{ksn}(16)$ & -3.7 & -12.8 & $1 \mathrm{nfw}$ & -12.2 & 0.6 \\
\hline $1 \mathrm{ksn}(16)$ & -3.7 & -12.8 & 1 nfu & -10.6 & 2.2 \\
\hline $1 \mathrm{ksn}(16)$ & -3.7 & -12.8 & $1 \mathrm{nfx}$ & -11.6 & 1.2 \\
\hline $1 \mathrm{ksn}(16)$ & -3.7 & -12.8 & 1 nfy & -12.1 & 0.7 \\
\hline $11 \operatorname{lpz}(200)$ & -3.3 & $-10.4^{25}$ & $1 \mathrm{mq} 5$ & -12.3 & -1.9 \\
\hline $1 \operatorname{lpz}(200)$ & -3.3 & -10.4 & $1 \mathrm{mq} 6$ & -13.8 & -3.4 \\
\hline $1 \operatorname{lpz}(200)$ & -3.3 & -10.4 & $1 \mathrm{nfw}$ & -12.2 & -1.8 \\
\hline $11 \mathrm{pz}(200)$ & -3.3 & -10.4 & 1nfu & -10.6 & -0.2 \\
\hline $11 \operatorname{lpz}(200)$ & -3.3 & -10.4 & $\operatorname{lnfx}$ & -11.6 & -1.2 \\
\hline
\end{tabular}




\begin{tabular}{|c|c|c|c|c|c|}
\hline$p d b(\text { water })^{a}$ & $\Delta G_{\text {wat }}^{b}$ & $\Delta G_{\text {lig1 }}^{c}$ & Ligand $2^{d}$ & $\Delta G_{\text {lig2 }}^{e}$ & $\Delta \Delta G_{l i g}^{f}$ \\
\hline $11 \mathrm{pz}(200)$ & -3.3 & -10.4 & 1nfy & -12.1 & -1.7 \\
\hline $1 \operatorname{lpg}(215)$ & -2.3 & $-9.7^{25}$ & $1 \mathrm{mq5}$ & -12.3 & -2.6 \\
\hline $1 \operatorname{lpg}(215)$ & -2.3 & -9.7 & $1 \mathrm{mq} 6$ & -13.8 & -4.1 \\
\hline $1 \operatorname{lpg}(215)$ & -2.3 & -9.7 & $1 \mathrm{nfw}$ & -12.1 & -2.4 \\
\hline $1 \operatorname{lpg}(215)$ & -2.3 & -9.7 & 1nfu & -10.6 & -0.9 \\
\hline $1 \operatorname{lpg}(215)$ & -2.3 & -9.7 & $\operatorname{lnfx}$ & -11.6 & -1.9 \\
\hline $1 \operatorname{lpg}(215)$ & -2.3 & -9.7 & 1nfy & -12.1 & -2.4 \\
\hline 1f0s(68) & 1.7 & $-10.6^{21}$ & $1 \mathrm{mq} 5$ & -12.3 & -1.8 \\
\hline 1f0s(68) & 1.7 & -10.6 & $1 \mathrm{mq} 6$ & -13.8 & -3.3 \\
\hline 1f0s(68) & 1.7 & -10.6 & $\operatorname{lnfw}$ & -12.1 & -1.6 \\
\hline 1f0s(68) & 1.7 & -10.6 & $1 \mathrm{nfu}$ & -10.6 & 0.0 \\
\hline 1f0s(68) & 1.7 & -10.6 & $1 \mathrm{nfx}$ & -11.6 & -1.0 \\
\hline 1f0s(68) & 1.7 & -10.6 & 1nfy & -12.1 & -1.5 \\
\hline 1ezq(115) & -2.4 & -12.3 & $11 \mathrm{pg}$ & -9.7 & 2.6 \\
\hline 1ezq(115) & -2.4 & -12.3 & 1lpk & -10.3 & 2.0 \\
\hline 1ezq(115) & -2.4 & -12.3 & $11 \mathrm{pz}$ & -10.4 & 1.9 \\
\hline 1ezq(115) & -2.4 & -12.3 & $1 x k a$ & -9.4 & 2.9 \\
\hline $1 \mathrm{ksn}(133)$ & -1.0 & -12.8 & $11 \mathrm{pg}$ & -9.7 & 3.1 \\
\hline $1 \mathrm{ksn}(133)$ & -1.0 & -12.8 & 1lpk & -10.3 & 2.5 \\
\hline $1 \mathrm{ksn}(133)$ & -1.0 & -12.8 & $11 \mathrm{pz}$ & -10.4 & 2.4 \\
\hline $1 \mathrm{ksn}(133)$ & -1.0 & -12.8 & $1 x k a$ & -9.4 & 3.4 \\
\hline $1 \mathrm{fOu}(6)$ & -1.8 & $-9.8^{21}$ & 1gi6 & $-8.5^{21}$ & 1.3 \\
\hline 1f0u(6) & -1.8 & -9.8 & $102 \mathrm{~m}$ & $-6.0^{26}$ & 3.8 \\
\hline $1 \mathrm{fOu}(6)$ & -1.8 & -9.8 & $102 p$ & $-6.6^{26}$ & 3.2 \\
\hline 1az8(638) & -2.5 & $-9.5^{27}$ & 1gi6 & -8.5 & 1.0 \\
\hline 1az8(638) & -2.5 & -9.5 & $102 \mathrm{~m}$ & -6.0 & 3.5 \\
\hline $1 \mathrm{az8}(638)$ & -2.5 & -9.5 & $102 p$ & -6.6 & 2.9 \\
\hline 1 bty (268) & -1.5 & $-7.3^{2}$ & 1gi6 & -8.5 & -1.3 \\
\hline 1 bty(268) & -1.5 & -7.3 & $102 \mathrm{~m}$ & -6.0 & 1.3 \\
\hline 1bty(268) & -1.5 & -7.3 & $102 p$ & -6.6 & 0.7 \\
\hline $1 \mathrm{c} 5 \mathrm{t}(325)$ & -1.1 & $-5.6^{28}$ & 1gi6 & -8.5 & -2.9 \\
\hline $1 \mathrm{c} 5 \mathrm{t}(325)$ & -1.1 & -5.6 & $102 \mathrm{~m}$ & -6.0 & -0.4 \\
\hline $1 \mathrm{c} 5 \mathrm{t}(325)$ & -1.1 & -5.6 & $102 p$ & -6.6 & -1.0 \\
\hline 1gi1(268) & -3.6 & $-7.4^{2}$ & 1gi6 & -8.5 & -1.1 \\
\hline 1gi1(268) & -3.6 & -7.4 & $102 \mathrm{~m}$ & -6.0 & 1.4 \\
\hline 1gi1(268) & -3.6 & -7.4 & $102 p$ & -6.6 & 0.8 \\
\hline
\end{tabular}




\begin{tabular}{|cccccc|}
\hline$p d b(\text { water })^{a}$ & $\Delta G_{\text {wat }}^{b}$ & $\Delta G_{\text {lig } 1}^{c}$ & Ligand $^{d}$ & $\Delta G_{\text {lig } 2}^{e}$ & $\Delta \Delta G_{\text {lig }}^{f}$ \\
\hline $1 \mathrm{o} 2 \mathrm{j}(705)$ & -1.6 & $-7.8^{26}$ & $1 \mathrm{gi6}$ & -8.5 & -0.7 \\
$1 \mathrm{o} 2 \mathrm{j}(705)$ & -1.6 & -7.8 & $1 \mathrm{o} 2 \mathrm{~m}$ & -6.0 & 1.8 \\
$1 \mathrm{o} 2 \mathrm{j}(705)$ & -1.6 & -7.8 & $1 \mathrm{o} 2 \mathrm{p}$ & -6.6 & 1.2 \\
$1 \mathrm{~b} 4 \mathrm{z}(455)$ & -4.2 & $-7.1^{29}$ & $1 \mathrm{~b} 40$ & -9.9 & -2.8 \\
$1 \mathrm{~b} 4 \mathrm{z}(455)$ & -4.2 & -7.1 & $1 \mathrm{~b} 58$ & -9.0 & -1.9 \\
$1 \mathrm{~b} 4 \mathrm{z}(455)$ & -4.2 & -7.1 & $1 \mathrm{qka}$ & -8.1 & -1.0 \\
$1 \mathrm{~b} 4 \mathrm{z}(455)$ & -4.2 & -7.1 & $2 \mathrm{olb}$ & -7.6 & -0.5 \\
$1 \mathrm{~b} 31(45)$ & -7.9 & $-8.0^{29}$ & $1 \mathrm{~b} 40$ & $-9.9^{30}$ & -1.9 \\
$1 \mathrm{~b} 31(45)$ & -7.9 & -8.0 & $1 \mathrm{~b} 58$ & $-9.0^{30}$ & -1.0 \\
$1 \mathrm{~b} 31(45)$ & -7.9 & -8.0 & $1 \mathrm{qka}$ & $-8.1^{30}$ & -0.1 \\
$1 \mathrm{~b} 31(45)$ & -7.9 & -8.0 & $2 \mathrm{olb}$ & $-7.6^{29}$ & 0.4 \\
$1 \mathrm{jet}(557)$ & -5.3 & $-9.9^{29}$ & $1 \mathrm{~b} 40$ & -9.9 & 0.0 \\
$1 \mathrm{jet}(557)$ & -5.3 & -9.9 & $1 \mathrm{~b} 58$ & -9.0 & 0.9 \\
$1 \mathrm{jet}(557)$ & -5.3 & -9.9 & $1 \mathrm{qka}$ & -8.1 & 1.8 \\
$1 \mathrm{jet}(557)$ & -5.3 & -9.9 & $2 \mathrm{olb}$ & -7.6 & 2.3 \\
\hline
\end{tabular}

${ }^{a} \mathrm{pdb}$ code and ID of the studied water in the $\mathrm{pdb} ;{ }^{b}$ calculated binding free energy of waters; ${ }^{c}$ experimental binding free energies of ligand in $\mathrm{pdb}$ code; ${ }^{d} \mathrm{pdb}$ code of structure with ligand displacing the water; ${ }^{e}$ experimental free energy of binding of ligand displacing the water; ${ }^{f} \Delta \Delta G_{\text {lig }}=\Delta G_{\text {lig2 } 2}-\Delta G_{\text {lig1 } 1}$. All $\Delta G$ values are in $k_{c a l ~ m o l}{ }^{-1}$.

\section{References and Notes}

(1) Burmeister, W. P.; Cusack, S.; Ruigrok, R. W. J. Gen. Vir., 1994, 75, 381-388.

(2) Katz, B. A.; Elrod, K.; Luong, C.; Rice, M .J.; Mackman, R. L.; Sprengeler, P. A.; Hataye, J. S. J.; Janc, J.; Link, J.; Litvak, J.; Rice, R. R. K.; Sideris, S.; Verner, E.; Young, W., J. Mol. Biol., 2001, 307, 1451-1486.

(3) Le Bonniec, B. F.; Guinto, E. R.; Esmon, C. T., J. Biol. Chem., 1992, 267, 6970-6976.

(4) Zheng, Y.; Bruice, T. C., Proc. Natl. Acad. Sci. USA, 1998, 95, 4158-4163.

(5) Holzer, C. T.; von Itzstein, M.; Jin, B.; Stewart, W. P.; Wu, W. Y., Glycoconjugate J., 1993, $10,40-44$.

(6) Kati, W. M.; Montgomery, D.; Carrick, R.; Gubareva, L.; Maring, C.; McDaniel, K.; 
Steffy, K.; Molla, A.; Hayden, F.; Kempf, D.; Kohlbrenner, W., Antimicrob. Agents Chemother., 2002, 46, 1014-1021.

(7) Wall, I. D.; Leach, A. R.; Salt, D. W.; Ford, M. G.; Essex, J. W., J. Med. Chem., 1999, $42,5142-5152$.

(8) Varghese, J. N.; Smith, P. W.; Sollis, S. L.; Blick, T. J.; Sahasrabudhe, A.; McKimmBreschkin, J. L.; Colman, P. M., Structure, 1998, 6, 735-746.

(9) Masukawa, M. K.; Kollman, P. A.; Kuntz, I. D., J. Med. Chem., 2003, 46, 5628-5637.

(10) Baldwin, E. T.; Bhat, T. N.; Gulnik, S.; Liu, B.; Topol, I. A.; Kiso, Y.; Mimoto, T.; Mitsuya, H.; Erickson, J. W., Structure, 1995, 3, 581-590.

(11) Lam, P. Y. S.; Jadhav, P. K.; Eyermann, C. J.; Hodge, C. N.; Ru, Y.; Bacheler, L. T.; Meek, J. L.; Otto, M. J.; Rayner, M. M.; Wong, Y. N.; Chang, C.; Weber, P. C.; Jackson, D. A.; Sharpe, T. R.; Erickson-Vitanen, S. Science, 1994, 263, 380-384.

(12) Hodge, N. C.; Aldrich, P. E.; Bacheler, L. T.; Chang, C-H.; Eyermann, C. J.; Garber, S.; Grubb, M.; Jackson, D. A.; Prabharkar, K. J.; Korant, B.; Lam, P. Y. S.; Maurin, M. B.; Meek, J. L.; Otto, M. J.; Rayner, M. M.; Reid, C.; Sharpe, T. R.; Linyee, S.; Winslow, D. L.; Erickson-Viitanen, S., Chem. Biol., 1996, 3, 301-314.

(13) Ala, P. J.; DeLoskey, R. J.; Huston, E. E.; Jadhav, P. K.; Lam, P. Y. S.; Eyermann, C. J.; Hodge, C. N.; Schadt, M. C.; Lewandowski, F. A.; Weber, P. C.; McCabe, D. D.; Duke, J. L.; Chang, C-H., J. Biol. Chem., 1998, 273, 12325-12331.

(14) Backbro, K.; Lowgren, S.; Osterlund, K.; Atepo, J.; Unge, T.; Hulten, J.; Bonham, N. M.; Schaal, W.; Karlen, A.; Hallberg, A., J. Med. Chem., 1997, 40, 898-902.

(15) Ala, P. J.; Huston, E. E.; Klabe, R. M.; Jadhav, P. K.; Lam, P. Y.; Chang, C. H., Biochemistry, 1998, 37, 15042-15049. 
(16) Alterman, M.; Andersson, H. O.; Garg, N.; Ahisen, G.; Lovgren, S.; Classon, B.; Danielson, U. H.; Kvarnstrom, I.; Vrang, L.; Unge, T.; Samuelsson, B.; Hallberg, A., J. Med. Chem., 1999, 42, 3835-3844,

(17) Pyring, D.; Lindberg, J.; Rosenquist, A.; Zuccarello, G.; Kvarnstrom, I.; Zhang, H.; Vrang, L.; Unge, T.; Classon, B.; Hallberg, A.; Samuelsson, B., J. Med. Chem., 2001, 44, 3083-3091.

(18) Alterman, M.; Bjorsne, M.; Muhlman, A.; Classon, B.; Kvarnstrom, I.; Danielson, H.; Markgren, P. O.; Nillroth, U.; Unge, T.; Hallberg, A.; Samuelsson, B., J. Med. Chem., 1998, $41,3782-3792$.

(19) Wawrzak, Z.; Sandalova, T.; Steffens, J. J.; Basarab, G. S.; Lundqvist, T.; Lindqvist, Y.; Jordan, D. B., Proteins, 1999, 35, 425-439.

(20) Chen, J. M.; Xu, S. L.; Wawrzack, Z.; Basarab, G. S.; Jordan, D. B., Biochemistry, 1998, $37,17735-17744$.

(21) Maignan, S.; Guilloteau, J. P.; Pouzieux, S.; Choi-Sledeski, Y. M.; Becker, M. R.; Klein, S. I.; Ewing, W.R.; Pauls, H. W.; Spada, A. P.; Mikol, V., J. Med. Chem., 2000, 43, 3226-3232.

(22) Adler, M.; Kochanny, M. J.; Ye, B.; Rumennik, G.; Light, D. R.; Biancalana, S.; Whitlow, M., Biochemistry, 2002, 41, 15514-15523.

(23) Maignan, S.; Guilloteau, J. P.; Choi-Sledeski, Y. M.; Becker, M. R.; Ewing, W.R.; Pauls, H. W.; Spada, A. P.; Mikol, V. J. Med. Chem., 2003, 46, 685-690.

(24) Guertin, K. R.; Gardner, C. J.; Klein, S. I.; Zulli, A. L.; Czekaj, M.; Gong, Y.; Spada, A. P.; Cheney, D. L.; Maignan, S.; Guilloteau, J. P.; Brown, K. D.; Colussi, D. J.; Chu, V.; Heran, C. L.; Morgan, S. R.; Bentley, R. G.; Dunwiddie, C. T.; Leadley, R. J.; Pauls, H. W. Bioorg. Med. Chem. Lett., 2002, 12, 1671-1674. 
(25) Matter, H.; Defossa, E.; Heinelt, U.; Blohm, P.; Schneider, D.; Muller, A.; Herok, S.; Schreuder, H.; Liesum, A.; Brachvogel, V.; Lonze, P.; Walser, A.; Al-Obeidi, F.; Wildgoose, P. J. Med. Chem., 2002, 45, 2749-2769.

(26) Katz, B. A.; Elrod, K.; Verner, E.; Mackman, R. L.; Luong, C.; Shrader, W. D.; Sendzik, M.; Spencer, J. R.; Sprengler, P. A.; Kolesnikov, A.; Tai, V. W. F.; Hui, H. C.; Breitenbucher, J. G.; Allen, D.; Janc, J. W., J. Mol. Biol., 2003, 329, 93-120.

(27) Quan, M. L.; Wexler, R. R., Curr. Top. Med. Chem., 2001, 1, 137-149.

(28) Katz, B. A.; Mackman, R.; Luong, C.; Radika, K.; Martelli, A.; Sprengeler, P. A.; Wang, J.; Chan, H.; Wong, L., Chem. Biol., 2000, 7, 299-312.

(29) Ladbury, J. E. Chem. Biol., 1996, 3, 973-980.

(30) Sleigh, S. H.; Seavers, P. R.; Wilkinson, A. J.; Ladbury, J. E.; Tame, J. R. H. J. Mol. Biol., 1999, 291, 393-415. 